\title{
Physical and electrical characterization of TexasPEG: An electrically conductive neuronal scaffold
}

\author{
William K. A. Sikkema ${ }^{1}$, Andrew B. Metzger ${ }^{1}$, Tuo Wang ${ }^{1}$, James M. Tour ${ }^{1,2,3}$ \\ ${ }^{1}$ Department of Chemistry, ${ }^{2}$ The NanoCarbon Center, ${ }^{3}$ Department of Material Science and Nanoengineering, Rice University, Houston, Texas, USA \\ E-mail:William K. A. Sikkema -William.sikkema@gmail.com; Andrew B. Metzger -Am86@rice.edu;Tuo Wang -Tuo.Wang@rice.edu; \\ *James M. Tour - Tour@rice.edu \\ *Corresponding author
}

Received:01 September $16 \quad$ Accepted: 16 February $17 \quad$ Published: 26 May 17

\begin{abstract}
Background: Graphene and its derivatives have been shown to be biocompatible and electrically active materials upon which neurons readily grow. The fusogen poly(ethylene glycol) (PEG) has been shown to improve outcomes after cervical and dorsal spinal cord transection. The long and narrow PEGylated graphene nanoribbon stacks (PEG-GNRs) with their $5 \mu \mathrm{m} \times 200 \mathrm{~nm} \times 10 \mathrm{~nm}$ dimensions can provide a scaffold upon which neurons can grow and fuse. We disclose here the extensive characterization data for the PEG-GNRs.

Methods: PEG-GNRs were chemically synthesized and chemically and electrically characterized.

Results: The average aspect ratio of the PEG-GNRs was determined to be $\sim 85$, which corresponds to a critical percolation value (the point where insulating material becomes conductive by addition of conductive particles) of $1 \%$. However, there was not a sharp increase in AC conductivity at frequencies relevant to action potentials.

Conclusion: A robust characterization of PEG-GNRs is discussed, though the precise origin of efficacy in improving outcomes following spinal cord transection is not known.

Key Words: Fusogen, graphene, nanoribbons, PEG

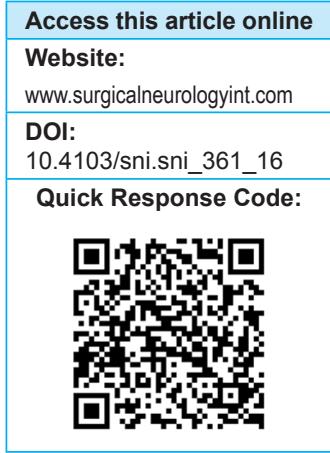

\section{INTRODUCTION}

Graphitic structures have been shown to electrically stimulate, physically support, and organize the three-dimensional (3D) structure of neurons. ${ }^{[2,6,10]}$ Many types of graphene have been used for spinal cord injury, ${ }^{[7,15,19]}$ however, to our knowledge, no one has used graphene nanoribbons (GNRs), despite the literature showing that GNRs positionally inform neurons grown in cell culture. ${ }^{[1]}$ In the context of the GEMINI spinal cord fusion protocol, ${ }^{[5]}$ we anticipated that GNRs could act as an electrical stop-gap to transmit electrical signals across the gap produced by sharp cervical cord transection. GNRs might further act as a scaffold for regrowth of neuronal processes. The scaffold could be especially useful if the GNRs could be aligned parallel to the spinal cord across the gap by either non-contact methods, such as electric fields, ${ }^{[17]}$ or teslaphoresis, ${ }^{[4]}$ or by contact methods that briefly separates the cut ends and causes shear forces to align the nanoribbons. ${ }^{[18]}$

This is an open access article distributed under the terms of the Creative Common Attribution-NonCommercial-ShareAlike 3.0 License, which allows others to remix, tweak, and build upon the work non-commercially, as long as the author is credited and the new creations are licensed under the identical terms.

For reprints contact: reprints@medknow.com

How to cite this article: Sikkema WK, Metzger AB, Wang T, Tour JM. Physical and electrical characterization of TexasPEG: An electrically conductive neuronal scaffold. Surg Neurol Int 2017;8:84.

http://surgicalneurologyint.com/Physical-and-electrical-characterization-ofTexasPEG:-An-electrically-conductive-neuronal-scaffold/ 
Pure poly(ethylene glycol) (PEG) can restore at least partial motor function in rodents by acting as a fusogen to seal blunt ends of neuronal processes and connect neurons across the gap. ${ }^{[10,12]}$ However, PEG applied shortly after surgery is likely rapidly cleared from the area because of its low molecular weight and high water solubility. To improve outcomes, it is necessary to include a component that will persist longer at the cut site to continue to stimulate reconnection, and PEG-GNRs might also serve as a useful agent to slow the loss of the PEG.

Due to their large physical size, high molecular weight $\left(\sim 10^{9} \mathrm{~g} / \mathrm{mol}\right)$ and high aspect ratio, GNRs might remain in the tissue much longer. In addition, their conductive properties might allow them to act as an electrical conduit to restore conduction through the fusion interface much more quickly. ${ }^{[9]}$ As a high aspect ratio graphene, GNRs might direct anisotropic neuronal growth longitudinally with the spinal cord axis. ${ }^{[1]}$ The synergy of PEG as an acute fusogen and PEG-GNRs as a long term repair scaffold might be the mechanism for the enhanced positive outcomes in spinal cord repair. ${ }^{[9]}$

This paper complements a sister paper ${ }^{[9]}$ by characterizing the PEG-GNRs, the clinically relevant mixture of PEG and PEG-GNRs, in addition to providing a rationale for the formulation used.

\section{MATERIALS AND METHODS}

PEG-GNR (TexasPEG): Multi-walled carbon nanotubes (MWCNTs) were obtained from EMD Merck (produced by Mitsui \& Co., lot no. 2699-64E) and were used as received. Tetrahydrofuran (THF) was dried over solid $\mathrm{KOH}$ for several days, degassed, and freshly distilled from sodium/benzophenone under a $\mathrm{N}_{2}$ atmosphere. All chemicals were purchased from Sigma-Aldrich unless otherwise specified. Thermogravimetric analysis (TGA) measurements were performed on a TA instruments Q-600 Simultaneous TGA/DSC. The temperature was ramped at $10^{\circ} \mathrm{C} / \mathrm{min}$ until $850^{\circ} \mathrm{C}$ under argon. For transmission electron microscopy (TEM, JEOL JEM 2100F) analysis, the PEG-GNRs were dispersed in water and drop cast onto a lacey carbon grid and allowed to dry for $6 \mathrm{~h}$. For scanning electron microscope (SEM) analysis, the PEG-GNRs were dispersed in o-dichlorobenzene, briefly sonicated in a bath sonicator, and deposited on a silicon wafer at an approximate density of 1 PEG-GNR per $500 \mu \mathrm{m}^{2}$, from which the solvent was evaporated on a heat plate at $<100^{\circ} \mathrm{C}$. The sample was imaged by an FEI Quanta 400 ESEM FEG instrument. Thirty images were taken in a direct line across the sample starting from a random location to minimize selection bias, and the resulting images were analysed with the aid of ImageJ. Conductivity measurements were performed with a home-built copper parallel plate dip-probe connected to a Hewlett-Packard 3577a Network Analyzer. Calibrations were performed using methanol and a short-circuit liquid metal standard.

$1.0 \mathrm{~g}$ of Mitsui MWCNTs was added to a $1 \mathrm{~L}$ oven-dried, nitrogen-purged, Schlenk flask; $500 \mathrm{~mL}$ of THF was added. $2.5 \mathrm{~mL}$ of eutectic $\mathrm{NaK}$ (1:3.3 by mass, 1:1.9 by mol) was added under nitrogen. The reaction mixture was stirred at room temperature for 3 days, until very few liquid droplets of $\mathrm{NaK}$ remained. The reaction was cooled in a dry ice/acetone bath to $-78^{\circ} \mathrm{C}$, and $30 \mathrm{~g}$ $(0.7 \mathrm{~mol})$ of gaseous ethylene oxide was added from a lecture bottle over $90 \mathrm{~min}$. The mixture was slowly brought to room temperature and stirred for 3 days. A mixture of $\mathrm{NaH}(20 \mathrm{mmol}, 0.53 \mathrm{~g})$ and propargyl bromide (20 mmol, $2.4 \mathrm{~g}$ ) suspended/dissolved in dry toluene was added to terminate the ethylene oxide polymerization. The reaction was quenched by adding $20 \mathrm{~L}$ of water, and the dark gray precipitate was collected via filtration on a $0.22 \mu \mathrm{m}$ polyethersulfone (PES) membrane. The dark gray precipitate was filtered through a polytetrafluoroethylene (PTFE) membrane $(0.45 \mu \mathrm{m})$, followed by crossflow filtration with a $50 \mathrm{kDa}$ MWCO PES filter to remove unbound polymer. The PEG-GNRs final product $(1.3 \mathrm{~g})$ was collected on a PTFE membrane $(0.45 \mu \mathrm{m})$, washed with DI water $(3 \times 100 \mathrm{~mL})$, ethanol $(3 \times 100 \mathrm{~mL})$, DI water $(3 \times 100 \mathrm{~mL})$, and dried under high vacuum overnight. The propargyl units were added to some of the termini for future peptide additions if desired. Before use, the PEG-GNRs were dispersed in PEG 600 with an IKA T25 digital Ultra-Turrax machine running at $1000 \mathrm{rpm}$ with an S25N-18G Dispersing element attachment. (0.5-1\% (w/v) by GNR concentration). The mixture was tightly sealed in a $50 \mathrm{~mL}$ conical vial and was sterilized by $120^{\circ} \mathrm{C}$ pressurized steam for $30 \mathrm{~min}$.

\section{RESULTS AND DISCUSSION}

Characterization of relevant physical and electrical properties was performed. First, to assess the amount of polymer covalently bound to the GNRs, TGA was performed. PEG decomposes fully by $400^{\circ} \mathrm{C}$, while the GNRs are stable under the temperatures tested. The PEG-GNRs are composed of 30\% PEG, while GNRs comprise the remaining 70\% [Figure 1].

By examination of the SEM and TEM images in Figure 2, one can see that the MWCNTs were indeed split into GNRs, displaying wavy patterns characteristic of GNRs and a much smaller persistence length than MWCNTs. The GNRs are in triangular stacks that result from unzipping several nanotubes in a MWCNT, where the largest tube makes the widest ribbon at the bottom, and the smaller tubes make increasingly thinner GNRs, stacked in order on top of each other [Figure 2d]. The GNRs are not individuals, but staked structures that do not easily exfoliate. 
The critical percolation concentration is determined by the aspect ratio of the conductive structures. This was calculated by measuring the lengths of individually dispersed PEG-GNRs on a silicon substrate of every PEG-GNR longer than $1 \mu \mathrm{m}$ [Figure 2a]. The average width and height of PEG-GNRs was measured at higher magnification under TEM. Dividing the distribution of lengths over the average height and width resulted in a histogram [Figure 3] that fit a normal logarithm plot with a standard deviation of 35 , and a number average of the aspect ratio was 85 . This average aspect ratio corresponds to a critical void fraction necessary for percolation of $1 \%$ in order for a conductive path to form. ${ }^{[8]}$

The percolation conductivity was measured from a concentration of $0.003 \%$ to $100 \%$ of PEG-GNRs in PEG at $1 \mathrm{kHz}$, as this is approximately the frequency of neuronal signals [Figure 4]. While there was not a dramatic change in conductivity at $\sim 1 \%$ as the average

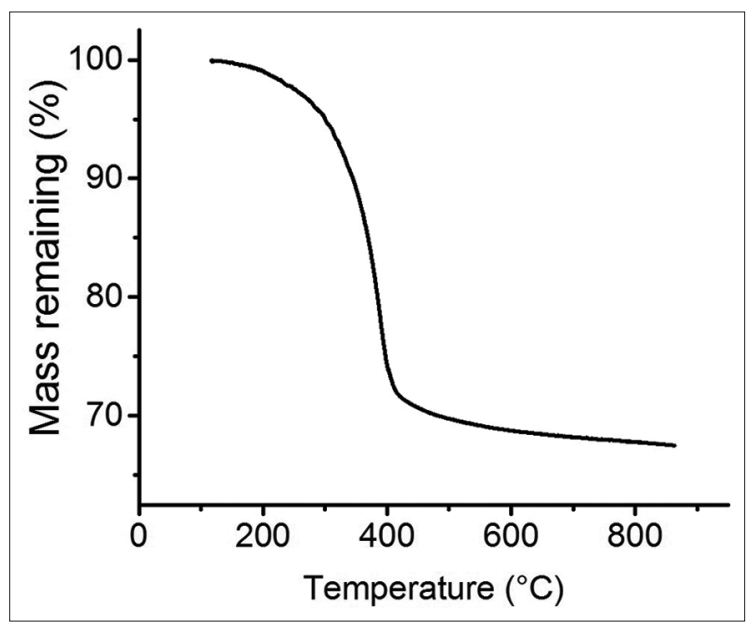

Figure I:Thermogravimetric analysis of PEG-GNRs under argon at a ramp $10^{\circ} \mathrm{C} / \mathrm{min}$, where PEG has completely decomposed before $400^{\circ} \mathrm{C}$, and the GNRs are stable past $800^{\circ} \mathrm{C}$

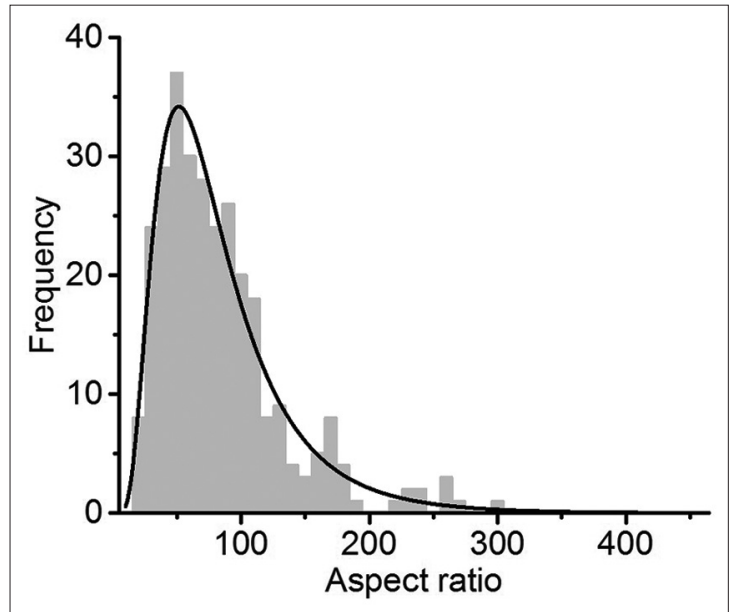

Figure 3: Measured histogram of aspect ratio of 300 PEG-GNRs as measured by TEM. Log normal fit gives a standard deviation of 36 , and the average aspect ratio is 85 aspect ratio predicted, the conductivity was an order of magnitude higher than the pure PEG. However, in a biological system, the PEG might diffuse away rapidly, leaving the PEG-GNRs behind in much higher concentration, allowing for the effective conductivity, and thus efficacy, of the PEG-GNR solution to rise over time.

\section{CONCLUSION}

In the sister paper, ${ }^{[9]}$ we have shown that the addition of PEG-GNRs to PEG dramatically increases the favourability of the outcomes following complete cervical spinal cord transection. In this paper, we have characterized the material as used. We anticipate further enhancements to the outcomes by ameliorating traumatically caused oxidative stress with our high-capacity, fast-acting antioxidants. ${ }^{[3,12,13,15]}$ For full

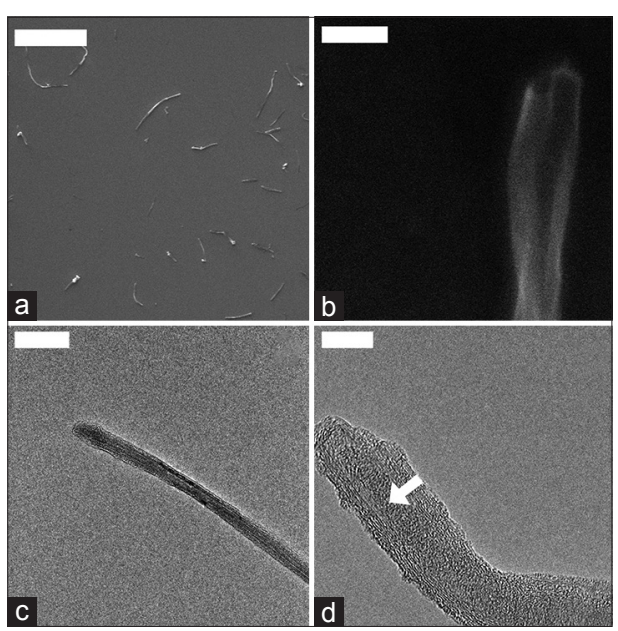

Figure 2: SEM and TEM analysis revealed the ribbon-like structure of the PEG-GNRs. (a) Individualized graphene nanoribbon stacks. Scale $=10 \mu \mathrm{m}$. (b) Open end of a large GNR structure. Scale $=200 \mathrm{~nm}$; (c) End of thin GNR. Scale = $100 \mathrm{~nm}$; (d) floppy end of a GNR stack, showing the triangular stack of GNRs. The white arrow shows the stack increasing in thickness. Scale $=10 \mathrm{~nm}$

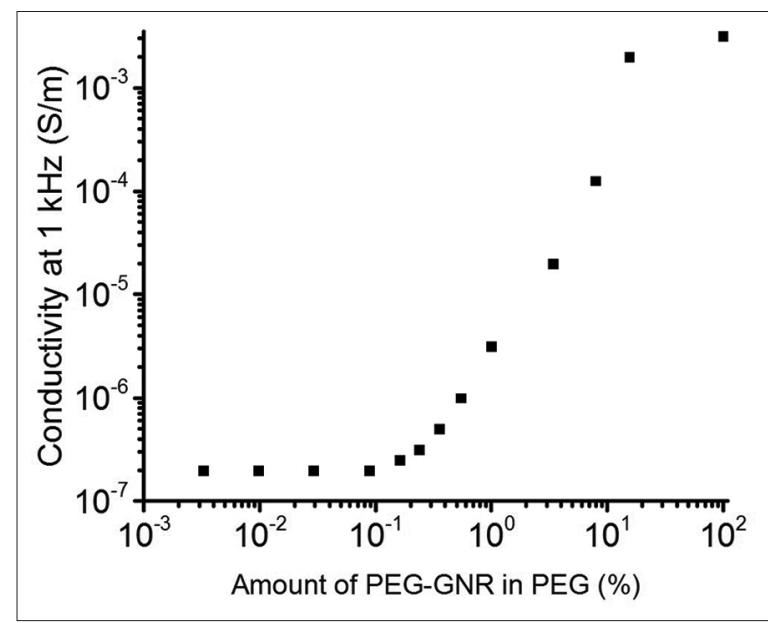

Figure 4: Experimentally measured conductivity of the PEG-GNR in PEG600 at varying concentrations 
effect, these can these be administered systemically and topically in the PEG/PEG-GNR mixture.

Financial support and sponsorship

We thank the Air Force Office of Scientific Research (FA9550-14-1-0111) for support.

\section{Conflicts of interest}

There are no conflicts of interest.

\section{REFERENCES}

I. Akhavan O, Ghaderi E. Differentiation of human neural stem cells into neural networks on graphene nanogrids. J Mater Chem B 2013;1:6291-30I.

2. Akhavan O, Ghaderi E, Abouei E, Hatamie S, Ghasemi E. Accelerated differentiation of neural stem cells into neurons on ginseng-reduced graphene oxide sheets. Carbon 2014;66:395-406.

3. Bitner BR, Marcano DC, Berlin JM, Fabian RH, Cherian L, Culver JC, et al. Antioxidant carbon particles improve cerebrovascular dysfunction following traumatic brain injury. J Neurotrauma 20I3;30:786-96.

4. Bornhoeft LR, Castillo AC, Smalley PR, Kittrell C, James DK, Brinson BE, et al. Teslaphoresis of carbon nanotubes. ACS Nano 2016;10:4873-8I.

5. Canavero S. The "Gemini” spinal cord fusion protocol: Reloaded. Surg Neurol Int 2015;6:18.

6. Fabbro A, Scaini D, Leon V, Vázquez E, Cellot G, Privitera G, et al. Graphene-based interfaces do not alter target nerve cells. ACS Nano 2015;10:615-23.

7. Fabbro A, Sucapane A, Toma FM, Calura E, Rizzetto L, Carrieri C, et al. Adhesion to carbon nanotube conductive scaffolds forces action-potential appearance in immature rat spinal neurons. PLoS One 2013;8:I-14.

8. Garboczi EJ, Snyder KA, Douglas JF, Thorpe MF. Geometrical percolation threshold of overlapping ellipsoids. Phys Rev E 1995;52:819-28.

9. Kim C, Sikkema WKA, Hwang I, Oh H, Kim UJ, et al. Spinal cord fusion with PEG-GNRs (TexasPEG): neurophysiological recovery in 24 hours in rats. Surg Neurol Int 2016;7:S632-6.

10. Kim CY. PEG-assisted reconstruction of the cervical spinal cord in rats: Effects on motor conduction at I h. Spinal Cord 2016; I:I-3.

II. Kim J, Park S, Kim YJ, Jeon CS, Lim KT, Seonwoo H, et al. Monolayer graphene-directed growth and neuronal differentiation OF mesenchymal stem cells. J Biomed Nanotechnol 20I5; I I:2024-33.

12. Luo J, Borgens R, Shi R. Polyethylene glycol immediately repairs neuronal membranes and inhibits free radical production after acute spinal cord injury. J Neurochem 2002;83:47I-80.

13. Marcano DC, Bitner BR, Berlin JM, Jarjour J, Lee JM, Jacob A, et al. Design of poly(ethylene glycol)-functionalized hydrophilic carbon clusters for targeted therapy of cerebrovascular dysfunction in mild traumatic brain injury. J Neurotrauma 2012;8: I-8.

14. Nilewski LG, Sikkema WKA, Tour JM, Kent TA. Carbon nanoparticles and oxidative stress: Could an injection stop brain damage in minutes? Nanomedicine 2015;10:1677-9.

15. Sahni D, Jea A, Mata JA, Marcano DC, Sivaganesan A, Berlin JM, et al. Biocompatibility of pristine graphene for neuronal interface. J Neurosurg Pediatr 20I3; I I:575-83.

16. Samuel ELG, Marcano DC, Berka V, Bitner BR, Wu G, Potter A, et al. Highly efficient conversion of superoxide to oxygen using hydrophilic carbon clusters. Proc Natl Acad Sci 2015; I I 2:2343-8.

17. Wang Z. Alignment of graphene nanoribbons by an electric field. Carbon $N$ Y 2009;47:3050-3.

18. Xu W, Lee T-W. Recent progress in fabrication techniques of graphene nanoribbons. Mater Horiz 2016;3:186-207.

19. Zhou K, Thouas GA, Bernard CC, Nisbet DR, Finkelstein DI, Li D, et al. Method to impart electro- and biofunctionality to neural scaffolds using graphene-polyelectrolyte multilayers. ACS Appl Mater Interfaces 20। 2;4:4524-3।. 